\title{
Erratum to: Nonseparable closed vector subspaces of separable topological vector spaces
}

\author{
Jerzy Ka̧kol ${ }^{1}$ • Arkady G. Leiderman² • \\ Sidney A. Morris ${ }^{3,4}$
}

\section{Erratum to: Monatsh Math DOI 10.1007/s00605-016-0876-2}

All our formal Theorems, Propositions, Corollaries, Examples are correct. One of our main results is

Theorem 2 Let $I$ be an index set and $E_{i}$ an lcs for each $i \in I$. If at least $\mathfrak{c}$ of the $E_{i}$ are not in $\mathfrak{V}(\mathbb{R})$, or equivalently do not have the weak topology, then the product $\prod_{i \in I} E_{i}$ has a nonseparable closed vector subspace.

However, some statements in the Abstract and elsewhere claim too much.

Thanks to e-mail from Stephen A. Saxon, we realized that the product $E^{\mathfrak{c}}$ may have a nonseparable closed vector subspace even when lcs $E$ has the weak topology. Take

The online version of the original article can be found under doi:10.1007/s00605-016-0876-2.

\footnotetext{
$凶$ Arkady G. Leiderman

arkady@math.bgu.ac.il

Jerzy Ka̧kol

kakol@amu.edu.pl

Sidney A. Morris

morris.sidney@gmail.com

1 Faculty of Mathematics and Informatics, A. Mickiewicz University, 61-614 Poznań, Poland

2 Department of Mathematics, Ben-Gurion University of the Negev, Beer Sheva, Israel

3 Faculty of Science and Technology, Federation University Australia, Ballarat, VIC 3353, Australia

4 School of Engineering and Mathematical Sciences, La Trobe University, Bundoora, VIC 3086, Australia
} 
$E$ to be the lcs of our Example 1; then this $E$ has the weak topology, is separable and contains a nonseparable closed vector subspace. Our erroneous claim appears after Problem 2, after Theorem 2, and in the Abstract. In particular, we have not given a complete answer to Problem 2.

Also, in the last sentence of the fifth paragraph of the Introduction, "a compact $X$ " should be replaced by "a separable compact $X$ ". 